\title{
Can Doping of Transition Metal Oxide Cathode Materials Increase Achievable Voltages with Multivalent Metals?
}

\author{
Daniel Koch $^{\mathrm{a}}$ and Sergei Manzhos ${ }^{\mathrm{b}}$
}

a. Department of Mechanical Engineering, National University of Singapore, Block EA \#07-08, 9 Engineering Drive 1, Singapore 117576. Email: koch.danielm@gmail.com

b. Centre Énergie Matériaux Télécommunications, Institut National de la Recherche Scientifique, 1650 boulevard Lionel-Boulet, Varennes QC J3X1S2 Canada. Email: sergei.manzhos@emt.inrs.ca

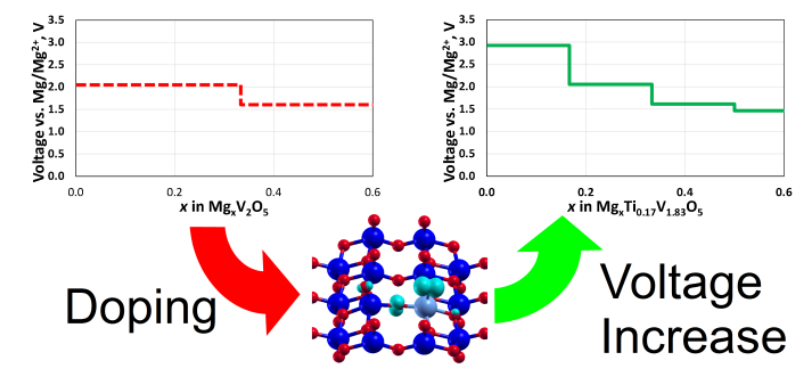

Synopsis: Density functional theory computations reveal how p-doping of transition metal oxide cathodes could increase voltages of multivalent batteries.

\begin{abstract}
We investigate from first principles the use of substitutional p-doping as a means to enhance the insertion energies of multivalent metals in transition metal oxides, and therefore the resulting voltages in an electrochemical cell, due to bandstructure modulation. Multivalent and earth-abundant metals such as magnesium or aluminium are attractive candidates to replace lithium in future high-performance secondary batteries with intercalation-type electrodes. Unfortunately, the achievable voltages obtained with this kind of elements still remain uncompetitively low. We study and compare the changes in insertion energetics (voltages) of single- and multivalent metals in semiconducting and insulating transition metal oxides upon substitutional p-doping with different metals, introducing different numbers of hole states. We use a single vanadium pentoxide monolayer as model system to study the effect of p-doping on achievable voltages and deduce general trends for transition metal oxides. Our investigations reveal the formation of $n$-hole polarons (with $n>1$ ) in form of oxygen dimers in $\mathrm{p}$-doped vanadia caused by localized $p$ holes on oxide ions in agreement with previous findings. We find that the oxygen dimer formation has an adverse effect on adsorption energetics compared to the single-hole case without dimerization. We
\end{abstract}


find an analogous oxygen dimerization in other TMOs with oxygen-dominated valence bands like molybdenum trioxide and titanium dioxide, while strained systems like trigonal nickelor titanium dioxide, or Mott-type systems like monoclinic vanadium dioxide with qualitatively different valence band composition do not exhibit oxygen dimerization with multi-hole doping. Our results demonstrate the advantages and limitations of TMO electrode p-doping and show a path to possible strategies to overcome detrimental effects.

\section{Introduction}

In 2018 , an estimated $56 \%$ of the global lithium (Li) consumption was accounted for by its use in secondary batteries, which is now the biggest sink for the sharply increasing global annual production $(85,000$ metric tons) of the relatively scarce $\mathrm{Li}$ metal (estimated global reserves of $\sim 14,000,000$ metric tons). ${ }^{1}$ Besides the resulting Li supply security concerns, the need for higher energy densities in mobile electrochemical energy storage, e.g. in electric vehicles, makes the use of multivalent metals like magnesium $(\mathrm{Mg})$ or aluminium (Al) as transient species in secondary batteries with intercalation-type cathodes interesting. This is due to the possibility of transferring more than one electron per migrating ion, hence potentially multiplying the specific capacity of the device. Insertion-type electrode material offer in general more stability compared to conversion reactions, since the topotactical insertion and release of metal ions into and from the electrodes does not require fundamental rearrangements in the crystal structure of the host, therefore providing greater reversibility and no disruptive changes in the physical properties of the electrodes (e.g. density). However, achievable voltages obtained with multivalent metals remain low, as the reaction enthalpy does not (necessarily) proportionally increase with the charge of the ion inserted into the cathode host (see eq. (1) below). Therefore, in order to obtain high energy densities with multivalent metals, the intercalation energies with the cathode material have to be increased. ${ }^{2}$ Possible avenues to achieve this goal are either the search for novel cathode materials or the modification of known insertion hosts to increase their binding strength with the multivalent solute.

Insertion-type cathode materials are commonly transition metal compounds such as phosphates, sulfides or, most prominently, oxides, due to the low-lying unoccupied $d$ states in these compounds relative to the Fermi level of the inserted metal in bulk, into which electrons are transferred during discharge. The frontier band structure of 
transition metal oxides (TMOs) used in batteries generally consists of low-lying, fully occupied oxygen $p$ states (with TM $d$ admixtures) and higher-lying, either un- or partially (depending on the compound stoichiometry) occupied TM $d$ states. Of the 792 binary transition metal oxides with the general stoichiometry $\mathrm{TM}_{\mathrm{x}} \mathrm{O}_{\mathrm{y}}$ currently included in the first-principles compound database Materials Project, ${ }^{3-5} 54 \%$ are predicted to exhibit a band gap of $0.1 \mathrm{eV}$ or more (and so do $63 \%$ of the 11,708 ternary oxides $\mathrm{A}_{\mathrm{w}} \mathrm{TM}_{\mathrm{x}} \mathrm{O}_{\mathrm{y}}$ with $\mathrm{A}$ being another element), making semiconducting and insulating TMOs the rule rather than the exception. The band gap in TMOs can generally have two origins: it is either due the energetic separation of bands with unlike character, namely low-lying oxygen- $p$ and higher-lying TM- $d$ bands (e.g. in charge-transfer insulators), or it is caused by the splitting of $d$ bands into low-lying occupied and high-lying unoccupied states (e.g. in Mott-Hubbard insulators).

Generally, the equilibrium voltage of a battery $V_{\text {eq }}$ is determined by the change in the molar Gibbs free energy $\Delta G$ (for the isothermal-isobaric conditions common in practical applications) between charged and discharged state per transferred charge

$$
V_{\mathrm{eq}}=-\frac{\Delta G}{z F}
$$

where $z$ is the valence electron number of the working metal and $F$ the Faraday constant. $\Delta G$ is the free energy of reaction for the discharge process happening in the electrochemical cell. For a Li-ion battery (LIB) with a bulk metal anode and TMO cathode for example, the overall reaction can be written as

$\mathrm{Li}_{(\mathrm{s})}+\mathrm{TMO}_{(\mathrm{s})} \rightarrow \mathrm{Li}\left[\mathrm{TMO}^{*}\right]_{(\mathrm{s})}$

where * denotes the deformed TMO cathode host upon metal insertion, and $\Delta G$ on the right is the free energy change of the reaction. To better understand the governing components of the reaction energy, the overall transformation above can be thought of as decomposed into elementary step reactions in the form of a Born-Haber cycle ${ }^{6}$ with individual reaction energies: ${ }^{7}$

$$
\begin{aligned}
& \mathrm{Li}_{(\mathrm{s})} \rightarrow \mathrm{Li}^{+}(\mathrm{g})+\mathrm{e}^{-} \\
& \Delta G_{1}=E_{\mathrm{coh}}+I E \\
& \mathrm{TMO}+\mathrm{e}^{-} \rightarrow \mathrm{TMO}^{-} \\
& \Delta G_{2}=W \\
& \mathrm{TMO}_{(\mathrm{s})}^{-} \rightarrow\left[\mathrm{TMO}^{*}\right]_{(\mathrm{s})}^{-} \\
& \Delta G_{3} \\
& \mathrm{Li}^{+}{ }_{(\mathrm{g})}+\left[\mathrm{TMO}^{*}\right]_{(\mathrm{s})}^{-} \rightarrow \mathrm{Li}\left[\mathrm{TMO}^{*}\right]_{(\mathrm{s})} \\
& \Delta G_{4}
\end{aligned}
$$

For other insertion cations, including polyvalent cations, a similar decomposition can be considered. The total reaction energy can be therefore written as $\Delta G=\Delta G_{1}+$ 
$\Delta G_{2}+\Delta G_{3}+\Delta G_{4}$. While the energies of certain reaction steps cannot be easily altered without changing the working metal, like $\Delta \mathrm{G}_{1}$ which depends on the metal's cohesive energy $\left(E_{\mathrm{coh}}\right)$ and ionization potential $(I E)$, and others are closely connected to the crystal structure of the host TMO, like the crystal structure deformation penalty $\Delta \mathrm{G}_{3}$, less invasive strategies exist for other energy components which can be used to tune the reaction energy and therefore cell voltage. In this work we want to specifically focus on the energy component $\Delta \mathrm{G}_{2}$, corresponding to the work function $W$ of the host TMO. A common approach to lower the work function of a material is through $\mathrm{p}$ doping. The rationale behind this functionalization strategy is rather straightforward: during battery cycling, electrons are transferred to the lowest unoccupied states at the conduction band minimum (which may fall into the bandgap upon occupation) of the electrode material taking up the transient metal ions (i.e. $\mathrm{Li}^{+}, \mathrm{Na}^{+}, \mathrm{Mg}^{2+}, \mathrm{Al}^{3+}$ etc.); the introduction of holes and therefore the creation of unoccupied states close to the valence band lowers the Fermi level of the material and enables the transfer of electrons into lower-lying states, generally leading to stronger binding by an increase of the band structure component of the total energy change.

It has been previously reported that p-doping of semiconducting materials can enhance their electrochemical activity by improving the solute-host binding, like in the monoelemental group 14 semiconductors $\mathrm{Si}$ and $\mathrm{Ge}$ doped with $\mathrm{Al}$ and $\mathrm{Ga}$, respectively, with a greatly enhanced affinity towards the insertion of $\mathrm{Li}, \mathrm{Na}$ and $\mathrm{Mg} .{ }^{8-}$ ${ }^{10}$ Substitutional doping of transition metal oxides has been reported as well, but the beneficial effects on the measured electrochemical performance have been mostly related to changes in the crystal rather than the electronic structure of the host material. ${ }^{11}$ In this work, we investigate from first principles the use of substitutional pdoping as a means to enhance the insertion energies of multivalent metals in transition metal oxides, and therefore the resulting voltages in an electrochemical cell, due to bandstructure modulation. We use to this end vanadium pentoxide $\left(\mathrm{V}_{2} \mathrm{O}_{5}\right)$ which is a semiconductor and a popular host for mono- and polyvalent cations. ${ }^{12-15}$ Theoretical studies on doped vanadia electrode materials have been limited to interstitial doping by metal intercalation (n-type), ${ }^{16}$ as well as hydrogenation ${ }^{17}$ and water cointercalation $^{18}$ which have been reported to effectively constitute $n$ - and p-doping, respectively. We also consider other oxides, including those with qualitatively different band compositions, for comparison. 


\section{Computational Approach}

To probe the effect of substitutional p-doping, we first studied the effects of dopant type, dopant concentration and dopant-adsorbate configurations on achievable voltages with a vanadium pentoxide $\left(\mathrm{V}_{2} \mathrm{O}_{5}\right)$ monolayer (slab thickness $\sim 5 \AA$, vacuum $\sim 20 \AA$ ) and adsorbed $\mathrm{Li}, \mathrm{Mg}$ and $\mathrm{Al}$. We chose this specific system since $\alpha-\mathrm{V}_{2} \mathrm{O}_{5}$ (space group Pmmn) is a frequently investigated candidate material for multivalent battery cathodes known for decades, ${ }^{19}$ with weakly bound nanosheets in a layered structure. These nanosheets can be exfoliated ${ }^{20}$ and have been shown to exhibit a competitive performance as Li-ion battery cathode material in experimental and theoretical studies $^{21-23}$ in terms of rate capabilities and capacities compared to bulk. The reduction of the system dimensionality with a single nanosheet instead of a bulk TMO further reduces complexity by neglect of weak interlayer interactions and steric effects exerted by neighboring layers on inserted (or adsorbed in the case of the 2D system) metal ions or axial oxygens, thus allowing a clearer view of effects of band structure modulation by doping. Substitutional dopants for $\mathrm{V}$ have been chosen to introduce one (Ti), two ( $\mathrm{Al})$, three $(\mathrm{Mg})$, or four $(\mathrm{Li})$ holes, while roughly matching the ionic radius of a $\mathrm{V}^{5+}$ ion; the adsorbed metal ions have been selected to compare one-, two-, and three-electron transfers. After the general trends have been established with a $\mathrm{V}_{2} \mathrm{O}_{5}$ monolayer, further bulk TMO systems with formally fully oxidized TM ions, molybdenum(VI) oxide $\left(\alpha-\mathrm{MoO}_{3}\right.$, space group Pnma) and titanium(IV) oxide (brookite and monolayer of trigonal $\mathrm{TiO}_{2}$, space groups $P b c a$ and $R-3 m$ ), as well as TMOs with significant $d$ populations in the valence band, monoclinic vanadium(IV) oxide $\left(\mathrm{VO}_{2}\right.$, space group $\left.P 2_{1} / c\right)$ and nickel(IV) oxide slabs (single layer of $\omega-\mathrm{NiO}_{2}$, space group $R-3 m$ ), were investigated to study the effects of $\mathrm{p}$-doping in bulk.

The computations were performed with density functional theory (DFT), employing the generalized gradient approximation functional of Perdew, Burke and Erznerhof $(\mathrm{PBE})^{24,25}$ as implemented in the program package SIESTA 4.1. ${ }^{26}$ The Hubbard correction ubiquitously used for TMOs was omitted in this work, as it was shown in previous investigations by our group ${ }^{27,28}$ that insertion energetics and electronic structure in these compounds can be matched using a localized basis set in form of pseudo-atomic orbitals on a final support ${ }^{29-31}$ for which the cutoff parameters are adjusted to reproduce experimental values. In this case the basis function cutoff radii were chosen such that cohesive energies of the main group elements were 
matched, as previously discussed in Ref. 27. The pseudo-atomic orbital confinement energy shifts were set to $0.02 \mathrm{Ry}$ for $\mathrm{Li}, 0.008 \mathrm{Ry}$ for $\mathrm{Mg}$ and $0.004 \mathrm{Ry}$ for $\mathrm{Al}$, while for the TMs and oxygen the cutoffs were chosen to match experimental insertion voltages in the corresponding TMOs with an energy shift of $0.001 \mathrm{Ry}$ for all species (for details see section 1 of the Supplementary Information). This scheme also correctly reproduces the appearance of localized gap states (see below). A double-zeta basis set was used with a real-space mesh cutoff of $400 \mathrm{Ry}$, and an energy-converged Monkhorst-Pack k-point $\operatorname{grid}^{32}$ with $\sim 30$ points $\AA$. Structure relaxations were performed using a conjugate gradient algorithm with a force tolerance of $0.02 \mathrm{eV} / \AA$ and a stress threshold of $0.01 \mathrm{GPa}$, partial occupancies were set with the MethfesselPaxton method ${ }^{33}$ and an electronic temperature of $500 \mathrm{~K}$. For layered compounds the cell volume was kept constant to prevent a collapse of the structure from pseudo-2D back to bulk. The spin polarization of individual atoms was obtained from the difference of atomic spin-up and spin-down Mulliken populations ${ }^{34}$ and was used to determine the number and positions of localized electrons and holes. Structure and spin density plots have been generated using the program XCrysDen v. 1.5.60. ${ }^{35}$

To compare the effect of substitutional doping, insertion/adsorption voltages were used as central performance metric. Neglecting entropic contributions and pressurevolume work for reactions in the solid state in eq. (1), as commonly done in the $a b$ initio literature ${ }^{36,37}$ on electrode materials, average voltages $U$ can be approximated as change in static DFT energy $E$ per transferred charge

$$
U(x, y)=-\frac{E\left(\mathrm{M}_{\mathrm{y}} \mathrm{TMO}\right)-E\left(\mathrm{M}_{\mathrm{x}} \mathrm{TMO}\right)-(y-x) E(\mathrm{M})}{(y-x) z F}
$$

$M$ denotes the inserted/adsorbed metal here with stoichiometric factors $x$ and $y$ at the onset of metalation and its end, respectively, $z$ is the valence electron number of $M$, and $F$ the Faraday constant. To construct a full voltage-capacity curve from first principles, stable phases that will sequentially form throughout the material have to be determined, since not all stoichiometries are thermodynamically stable and would rather disproportionate into the two nearest compositions on the convex hull in the formation energy-composition diagram of the M-TMO system. To obtain this convex hull, formation energies relative to the pristine and fully metalated phases $E_{\mathrm{f}}$ of all computed stoichiometries are obtained by the following expression: 


$$
E_{\mathrm{f}}\left(\mathrm{M}_{\mathrm{x}} \mathrm{TMO}\right)=E\left(\mathrm{M}_{\mathrm{x}} \mathrm{TMO}\right)-\frac{x}{x_{\mathrm{f}}} E\left(\mathrm{M}_{\mathrm{x}_{\mathrm{f}}} \mathrm{TMO}\right)-\left(1-\frac{x}{x_{\mathrm{f}}}\right) E(\mathrm{TMO})
$$

where $x_{\mathrm{f}}$ is the stoichiometric coefficient for the final state of charge. Using eq. (2), the approximate voltage-capacity curve can be constructed as sequence of stable phase formations throughout the whole material until the next stable phase with higher metal concentration is formed (two-step mechanism).

\section{Adsorption of Mono- and Multivalent Metals on a Vanadium Pentoxide}

\section{Monolayer}

Computations on the $\mathrm{V}_{2} \mathrm{O}_{5}$ nanosheet system were carried out in a simulation cell containing six formula units, as shown in Figure 1. Two inequivalent stable adsorption sites were identified and will be denoted in the following as $\mathrm{H} 1$ and $\mathrm{H} 2$ in accordance to previous reports. ${ }^{21}$ For all adsorbed species the $\mathrm{H} 1$ site, coordinated by four axial oxide ions above or below the layer, is found to be more stable than the $\mathrm{H} 2$ site, fourfold coordinated by equatorial oxide ions connecting two or three vanadium centers. The structure can be thought of as a shifted parallel arrangement of $\mathrm{V}_{2} \mathrm{O}_{5}$ "ladders" in $a$ direction, with $\mathrm{V}-\mathrm{O}-\mathrm{V}$ fragments in $c$ direction as "rungs". This terminology is common in the literature ${ }^{38,39}$ and will be used in the following to describe electronic structure and magnetic couplings in metalated $\mathrm{V}_{2} \mathrm{O}_{5}$.

Possible reasons for the preference of $\mathrm{H} 1$ over $\mathrm{H} 2$ sites are the greater flexibility of

(a)

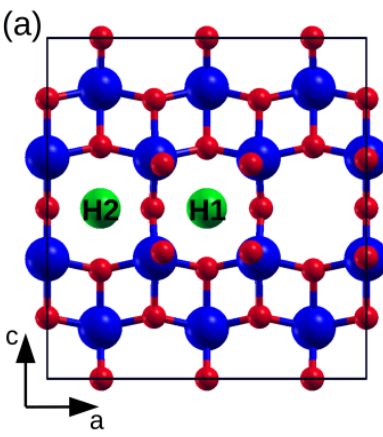

(b)

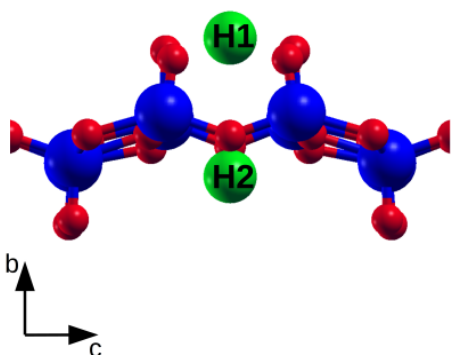

Figure 1. Simulation cell of the $\mathrm{V}_{2} \mathrm{O}_{5}$ nanosheet in top (a) and side (b) view. Vanadium atoms are indicated as blue, oxygen atoms as red spheres. The positions of two non-identical adsorption sites are indicated in green and labelled as $\mathrm{H} 1$ and $\mathrm{H} 2$. 
the axial oxide ions (bound only to one cation, hence lower energy penalty for displacement) and the larger separation between adsorbed and structural (vanadium) cations, decreasing their Coulombic repulsion. Electron transfer to $\mathrm{V}_{2} \mathrm{O}_{5}$ from the adsorbed working metals leads to localized charges on $\mathrm{V}$ (and $\mathrm{O}$ ) centers and the formation of small polarons ${ }^{40}$ by the distortion of the host structure, mainly an increase in the $\mathrm{V}-\mathrm{O}-\mathrm{V}$ angle on the rungs. With increasing degree of metalation, additional adsorbate atoms arrange in a way that maximizes their mutual separation in order to minimize the Coulombic interaction between the cations, an effect especially pronounced for the nanosheet system, as the cation charge shielding by oxide anions is reduced in the $2 \mathrm{D}$ slab.

\section{Lithium: Single-Electron Donation Mechanism}

Upon $\mathrm{Li}$ adsorption on the $\mathrm{V}_{2} \mathrm{O}_{5}$ nanosheet, one electron is initially donated to four $\mathrm{V}$ centers of which each carries a spin polarization of approximately $0.25 \mu_{\mathrm{B}}$ coupled by $\mathrm{O} p$ orbitals via superexchange interactions, as can be seen from the spin density in Figure 2(a). The density of states in Figure 2(a) shows the formation of a mid-gap state, even without the application of a Hubbard- $U$ correction normally necessary to observe this effect (specifically) with plane wave codes. The initial voltage of $3.35 \mathrm{eV}$ is slightly higher than in previous theoretical investigations of the $\mathrm{V}_{2} \mathrm{O}_{5}$ single layer, ${ }^{21}$, 22 although it should be mentioned that in those works a plane-wave basis with PBE without a correction for strong on-site repulsion was used, leading erroneously to delocalized electrons and occupied states at the conduction band minimum, possibly underestimating the binding energy. This is further supported by the good agreement of our calculation with the experimentally obtained low-concentration voltage plateau at $3.4 \mathrm{~V}$ for the cycling of $\mathrm{V}_{2} \mathrm{O}_{5}$ nanosheets against $\mathrm{Li}^{20}$ 
Further Li adsorption is computed to take place at two more distinct voltage plateaus at 3.02 and $2.53 \mathrm{~V}$, as shown in Figure 3, up to a final state of charge of $\mathrm{Li}_{2} \mathrm{~V}_{2} \mathrm{O}_{5}$, which corresponds to a gravimetric capacity of $294 \mathrm{mAh} / \mathrm{g}$. This agrees reasonably well with the experimentally observed two additional plateaus at 3.2 and $2.3 \mathrm{~V}$ and a final state of charge at $290 \mathrm{mAh} / \mathrm{g} .{ }^{20}$ The charge distribution in the form of spin densities for higher Li concentrations is shown in panels (b) and (c) of Figure 2. At a stoichiometry of $\mathrm{LiV}_{2} \mathrm{O}_{5}$ each $\mathrm{V}$ center carries a charge of approximately $-0.5|e|$, ferromagnetically coupled along the rungs. This type of quarter-filled spin-ladder system has been reported in the literature to exhibit antiferromagnetic coupling between the rungs, ${ }^{41,42}$ in part seen in Figure 2, but it shall be mentioned that the restricted simulation cell size of twelve $\mathrm{V}$ centers, corresponding to six rungs and only

(a)
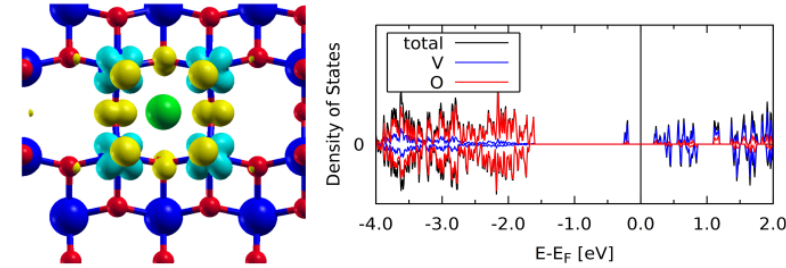

(b)
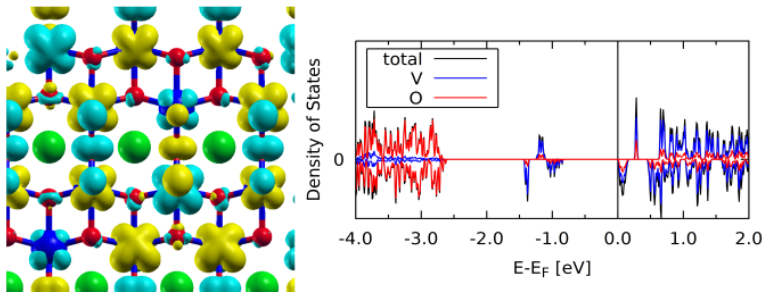

(c)
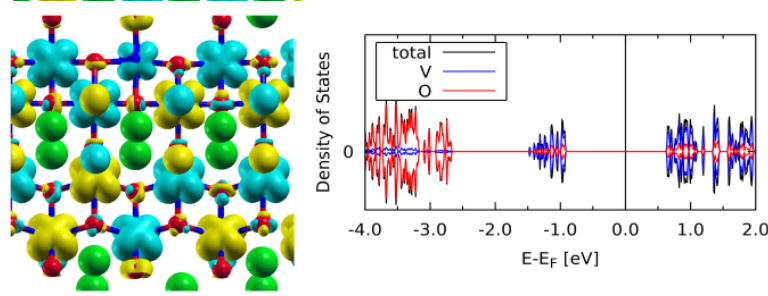

Figure 2. Spin densities (left) and densities of states (right) of (a) $\mathrm{Li}_{0.17} \mathrm{~V}_{2} \mathrm{O}_{5}$, (b) $\mathrm{LiV}_{2} \mathrm{O}_{5}$, and (c) $\mathrm{Li}_{2} \mathrm{~V}_{2} \mathrm{O}_{5}$. $\mathrm{Up}$-spin density is represented in light blue, down-spin density in yellow (isosurface value $0.005 e / \AA^{-3}$ ), while vanadium, oxygen, and lithium atoms as blue, red, and green spheres, respectively. Up- and down-spin densities of states are shown with positive and negative sign, respectively; the vanadium-projected density of states is plotted in blue, oxygen-projected in red, and the full one in black. 
three distinct rungs per ladder, does not allow for a fully antiferromagnetic coupling due to symmetry reasons. The charge ordering obtained with $\mathrm{PBE}$ for $\mathrm{LiV}_{2} \mathrm{O}_{5}$ corresponds to the high-temperature $(T>34 \mathrm{~K})$ charge ordering that has been found experimentally for $\mathrm{NaV}_{2} \mathrm{O}_{5}$, while for lower temperatures a charge ordering with half of the $\mathrm{V}$ centers occupied with one donated electron is found in experiments. ${ }^{39}$ Similarly to our $\mathrm{LiV}_{2} \mathrm{O}_{5}$ cell, the antiferromagnetic coupling between adjacent $\mathrm{V}$ centers in the half-filled spin ladder compound $\mathrm{Li}_{2} \mathrm{~V}_{2} \mathrm{O}_{5}$ cannot be achieved due to the simulation cell size, but the favorable occurrence of dissimilarly spin-polarized V(IV) centers is obtained nevertheless.

\section{Multivalent Cation Adsorption: Magnesium and Aluminium}

The computed voltages for the adsorption of the multivalent metals $\mathrm{Mg}$ and $\mathrm{Al}$ are lower than for $\mathrm{Li}$, and the ones obtained with $\mathrm{Al}$ anon lower than for $\mathrm{Mg}$, due to the higher transferred charge per atom (cf. eq. (2)) as can be seen in the voltage-capacity curve in Figure 3, where the low-concentration voltages for $\mathrm{Mg}$ and $\mathrm{Al}$ are $2.05 \mathrm{~V}$ (again slightly higher than the voltage of $1.74 \mathrm{~V}$ obtained previously with plane-wave $\mathrm{PBE}^{21}$ ) and $1.13 \mathrm{~V}$, respectively. Similarly to low Li concentrations, the two and three electrons donated by $\mathrm{Mg}$ and $\mathrm{Al}$, respectively, occupy a single $\mathrm{V}-\mathrm{O}-\mathrm{V}$ rung each. The corresponding spin densities of $\mathrm{Mg}_{0.17} \mathrm{~V}_{2} \mathrm{O}_{5}$ and $\mathrm{Al}_{0.17} \mathrm{~V}_{2} \mathrm{O}_{5}$ are shown in Figure 4.

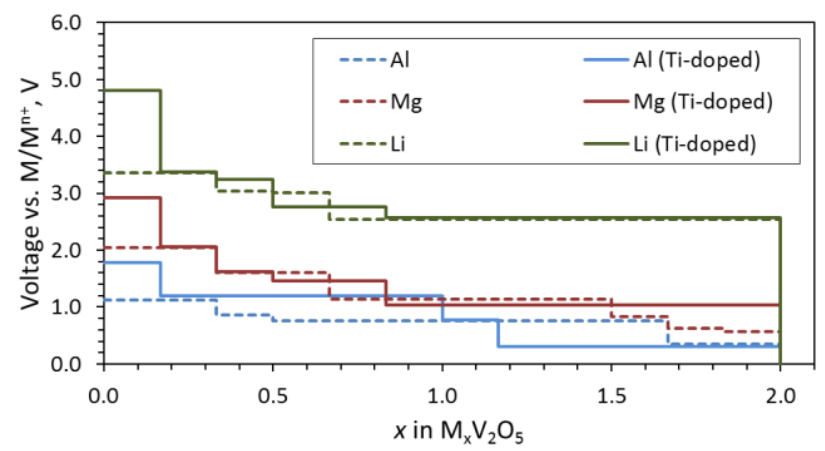

Figure 3. Computed voltage-capacity curve for the $\mathrm{Li}$ (green), $\mathrm{Mg}$ (red), and $\mathrm{Al}$ (blue) adsorption on a pristine (dashed line) and Ti-doped (solid line) $\mathrm{V}_{2} \mathrm{O}_{5}$ nanosheet. 
In the case of $\mathrm{Mg}$ adsorption, the two electrons are localized on adjacent rungs of the same ladder, with an antiferromagnetic coupling computed to be energetically more stable, while for Al the two rungs next to the adsorbate and one on a neighboring ladder are occupied all with collinear spin alignment; the DOS shows one distinct V mid-gap state per transferred electron (Figure 4). At higher metal concentrations exceeding a 0.5-electron donation mechanism, the electrons start to localize on individual $\mathrm{V}$ centers again. For stable phases of $\mathrm{Mg}_{\mathrm{x}} \mathrm{V}_{2} \mathrm{O}_{5}$ and $\mathrm{Al}_{\mathrm{x}} \mathrm{V}_{2} \mathrm{O}_{5}$ with $x>1$ and $x>0.67$, respectively, $\mathrm{V}$ centers with two or three localized electrons start to occur, while retaining the antiferromagnetic ordering of the electrons between different centers that has been found for $\mathrm{Li}_{2} \mathrm{~V}_{2} \mathrm{O}_{5}$ (see Figure 2). As shown in Figure 3, the occupation of all adsorption sites with $\mathrm{Mg}$ and $\mathrm{Al}$ is predicted to be thermodynamically stable, leading to final state of charge stoichiometries of $\mathrm{Mg}_{2} \mathrm{~V}_{2} \mathrm{O}_{5}$ and $\mathrm{Al}_{2} \mathrm{~V}_{2} \mathrm{O}_{5}$ (corresponding to gravimetric capacities of $588 \mathrm{mAh} / \mathrm{g}$ and $882 \mathrm{mAh} / \mathrm{g}$ respectively). The final discharge voltages for the $\mathrm{Mg}$ and $\mathrm{Al}$ adsorption drop to 0.57 $\mathrm{V}$ and $0.34 \mathrm{~V}$, respectively. It should be noted that while $\mathrm{Mg}_{2} \mathrm{~V}_{2} \mathrm{O}_{5}$ mostly retains the

(a)
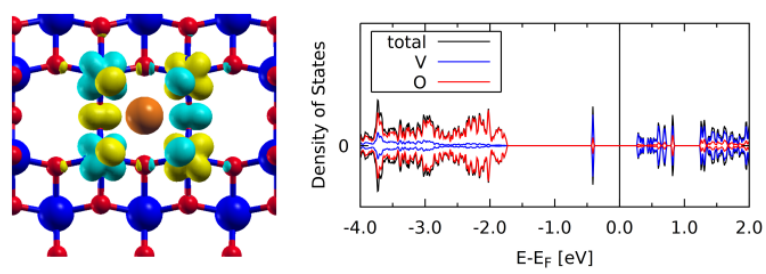

(b)
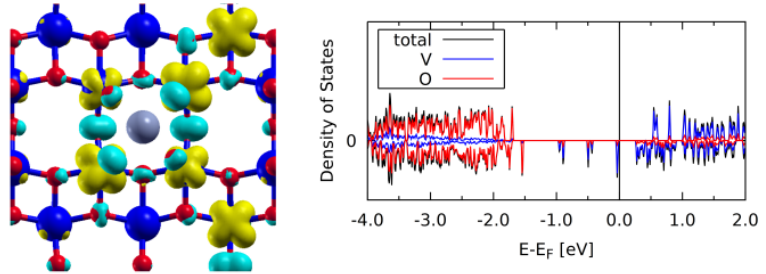

Figure 4. Spin densities (left) and densities of states (right) of (a) $\mathrm{Mg}_{0.17} \mathrm{~V}_{2} \mathrm{O}_{5}$, and (b) $\mathrm{Al}_{0.17} \mathrm{~V}_{2} \mathrm{O}_{5}$. Up-spin density is represented in light blue, down-spin density in yellow (isosurface value $0.005 e / \AA^{-3}$ ), while vanadium, oxygen, magnesium, and aluminium atoms as blue, red, orange, and grey spheres, respectively. Up- and downspin densities of states are shown with positive and negative sign, respectively; the vanadium-projected density of states is plotted in blue, oxygen-projected in red, and the full one in black. 
initial nanosheet structure, severe structural distortions start to occur for $\mathrm{Al}_{2} \mathrm{~V}_{2} \mathrm{O}_{5}$. This might indicate the onset of a more favorable conversion-type reaction mechanism for a 3-electron transfer reaction. This is further supported by the observed preference of conversion in cases where $\mathrm{V}$ (II) centers are formed in $\mathrm{VO}_{2}$, as computed by Canepa et al., ${ }^{43}$ while the formation of $\mathrm{V}(\mathrm{III})$ centers in $\mathrm{V}_{2} \mathrm{O}_{5}$ preferentially occurs via an insertion mechanism for $\mathrm{Mg}$ and Ca. Analogously, the reduction of $\mathrm{V}(\mathrm{V})$ to $\mathrm{V}(\mathrm{II})$ in $\mathrm{Al}_{2} \mathrm{~V}_{2} \mathrm{O}_{5}$ might favor a conversion reaction of the nanosheet instead of adsorption, but an in-depth analysis of the mechanistic preferences of the (2D) $\mathrm{V}_{2} \mathrm{O}_{5}$ structure is beyond the scope of this work. However, the intermediate $\mathrm{Al}_{1.67} \mathrm{~V}_{2} \mathrm{O}_{5}$ shows a good structure retention of the vanadium pentoxide monolayer, and the spin densities with antiferromagnetic coupling between $\mathrm{V}$ centers and densities of states for $\mathrm{Mg}_{2} \mathrm{~V}_{2} \mathrm{O}_{5}$ and $\mathrm{Al}_{1.67} \mathrm{~V}_{2} \mathrm{O}_{5}$ are shown in Figure 5 for comparison.

\section{Substitutional p-Doping of a Vanadium Pentoxide Monolayer}

For substitutional doping of $\mathrm{V}_{2} \mathrm{O}_{5}$, one vanadium atom in the simulation cell was

(a)

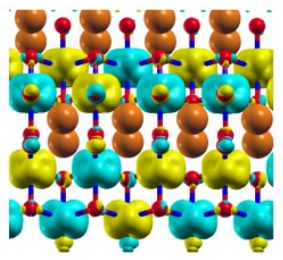

(b)

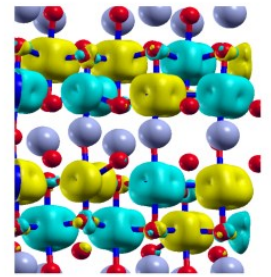

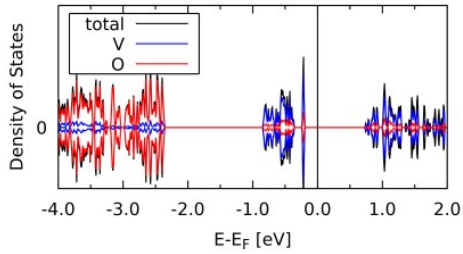

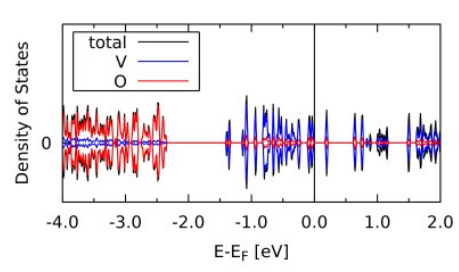

Figure 5. Spin densities (left) and densities of states (right) of (a) $\mathrm{Mg}_{2} \mathrm{~V}_{2} \mathrm{O}_{5}$, and (b) $\mathrm{Al}_{1.67} \mathrm{~V}_{2} \mathrm{O}_{5}$. Up-spin density is represented in light blue, down-spin density in yellow (isosurface value $0.01 \AA^{-3}$ ), while vanadia, oxygen, magnesium, and aluminium atoms as blue, red, orange, and grey spheres, respectively. Up- and downspin densities of states are shown with positive and negative sign, respectively; the vanadium-projected density of states is plotted in blue, oxygen-projected in red, and the full one in black. 
replaced by $\mathrm{Ti}, \mathrm{Al}, \mathrm{Mg}$ or $\mathrm{Li}$, resulting in a stoichiometry of $\mathrm{M}_{0.17} \mathrm{~V}_{1.83} \mathrm{O}_{5}$ (with $M$ being the dopant). For the single-hole doping with $\mathrm{Ti}$, the electron deficiency occurs mainly on the surrounding ions, as can be seen from the spin density and DOS in Figure 6(a), with most of the hole localized on the oxygen center axially bound to $\mathrm{Ti}$ (spin of $\sim 0.85 \mu_{\mathrm{B}}$ ), possibly due to the better charge compensation from the neighboring vanadium centers for the equatorial oxygen ligands of the Ti coordination polyhedron. The localized hole state and Ti substitution leads to a small polaron with an increased V-O-Ti rung angle of $178^{\circ}$ (142 ${ }^{\circ}$ for $\left.\mathrm{V}-\mathrm{O}-\mathrm{V}\right)$, and an elongated Ti-O bond ( $0.2 \AA$ increase compared to $\mathrm{V}-\mathrm{O})$.

For double- and triple-hole formations with $\mathrm{Al}$ and $\mathrm{Mg}$ the local lattice deformation becomes more severe, with a distinct dimerization of the axial oxygen center bound to the dopant and the equatorial one on the V-O-M rung. The distance between these oxygen centers reduces from $2.74 \AA$ in the pristine case to $1.48 \AA$ and $1.36 \AA$ in the double- and triple-hole case, respectively, due to the formation of $\mathrm{O}_{2}{ }^{2-}$ (exp. bond length $1.48 \AA^{44}$ ) and $\mathrm{O}_{2}^{-}$ions (exp. bond length $1.28 \AA^{45}$ ). This is in agreement with the double-hole-induced oxygen dimerization in TMOs $\left(\mathrm{TiO}_{2}, \mathrm{~V}_{2} \mathrm{O}_{5}\right.$ and $\left.\mathrm{MoO}_{3}\right)$ previously reported by Chen et $a l .{ }^{46}$ In their work they provide a mechanistic explanation for the dimerization of oxygen in strongly correlated oxides: the localized unpaired electrons (hole states) on two oxygen centers (in proximity of the dopant) are lowered in energy by occupying $\mathrm{O}_{2}$ molecular states formed by the $\mathrm{O}$ dimer polaron, while the unoccupied states get shifted up in energy, leading to an overall stabilization of the system. Here, we observe a similar effect for three and four holes. The latter case was probed by Li doping of $\mathrm{V}_{2} \mathrm{O}_{5}$ and led to a complete reduction of the oxide ions and the formation of volatile molecular oxygen and decomposition of the monolayer, which is why it will be further omitted in this discussion. The formation of occupied and unoccupied localized states on oxygen in $\mathrm{Al}-$ and $\mathrm{Mg}$-doped $\mathrm{V}_{2} \mathrm{O}_{5}$ is shown in the DOS and spin densities in Figure 6(b) and (c).

The doped monolayer was found to be stabilized through oxygen dimerization by $0.72 \mathrm{eV} /$ dopant and $2.15 \mathrm{eV} /$ dopant in the two- and three-hole case, respectively, compared to the doped system in which the O-O dimerization was artificially suppressed. In Table 1 the low-concentration voltage changes of the doped compared to the pristine nanosheet are compared for the dimerized and non-dimerized cases (where applicable). Due to its low stabilization energy, the oxygen dimer in Al-doped 
Table 1. Computed adsorption voltages of $\mathrm{Li}, \mathrm{Mg}$, and $\mathrm{Al}$ on Ti-, Al-, and Mg-doped $\mathrm{V}_{2} \mathrm{O}_{5}$ monolayers against bulk metal ( $\mathrm{Li}, \mathrm{Mg}$, and $\mathrm{Al}$, respectively). For two- and three-hole doping, voltages with and without the formed oxygen dimer are compared and the thermodynamically more stable case highlighted in bold. The values in parentheses denote the change in voltage compared to the pristine $\mathrm{V}_{2} \mathrm{O}_{5}$ monolayer.

\begin{tabular}{|c|c|c|c|c|c|}
\hline \multirow[b]{2}{*}{ adsorbate } & \multirow{2}{*}{$\begin{array}{c}T i_{0.17} V_{1.83} O_{5} \\
U[V]\end{array}$} & \multicolumn{2}{|c|}{$A l_{0.17} V_{1.83} O_{5}$} & \multicolumn{2}{|c|}{$M g_{0.17} V_{1.83} O_{5}$} \\
\hline & & $U($ dimer $)[V]$ & $U[V]$ & $U$ (dimer) $[V]$ & $U[V]$ \\
\hline$L i$ & $4.80(+1.47)$ & $3.42(+0.08)$ & $4.07(+0.73)$ & $3.76(+0.43)$ & $2.54(-0.80)$ \\
\hline$M g$ & $2.92(+0.88)$ & $2.09(+0.05)$ & $3.43(+1.39)$ & $2.26(+0.22)$ & $2.31(+0.27)$ \\
\hline$A l$ & $1.78(+0.71)$ & $1.15(+0.08)$ & $2.01(+0.94)$ & $1.28(+0.21)$ & $1.69(+0.62)$ \\
\hline
\end{tabular}

$\mathrm{V}_{2} \mathrm{O}_{5}$ is not stable under the adsorption of $\mathrm{Li}, \mathrm{Mg}$, or $\mathrm{Al}$, and the dissociation of the $\mathrm{O}_{2}{ }^{2-}$ anion and occupation of the two holes on oxygen centers by the donated electrons is thermodynamically more stable. It should be noted that this is only true for adsorbed metal ions in the vicinity of the dopant, as the electron-polaron is stabilized by the adsorbed metal ion and polaron hopping is restricted, hence an electron-hole separation occurs at large adsorbate-dopant distances when the energy penalty for polaron-ion separation exceeds the energy gain by electron-hole combination, resulting in lower voltages; for details see section 2 of the Supplementary Information. While this is not supposed to be problematic for systems with high ionic diffusivities (like the isolated $\mathrm{V}_{2} \mathrm{O}_{5}$ monolayer), where binding site preference is expected to be thermodynamically governed, a low accessibility of the insertion site in strongly correlated TMO materials could cause voltage losses. Here we only consider the configurations with highest binding energies.

In the case of $\mathrm{Mg}$ doping, the dimer is stable upon Li adsorption (since two holes remain for dimerization), while the dissociation of the $\mathrm{O}_{2}{ }^{-}$ion upon $\mathrm{Mg}$ adsorption is only about $0.1 \mathrm{eV}$ more stable than the dimer case. Restriction of the $\mathrm{O}-\mathrm{O}$ dimer bond length allows to compute approximate dissociation barriers, but these were found at very small values in the chosen test cases $\left(<0.03 \mathrm{eV}\right.$ for $\mathrm{Mg} @ \mathrm{MgV}_{11} \mathrm{O}_{30}$ and $\left.\mathrm{Mg} @ \mathrm{AlV}_{11} \mathrm{O}_{30}\right)$, and the dimerization process is reversible at standard operation conditions. Overall, the double and triple hole-induced oxygen dimerization reduces the accessible voltages by lowering the energy of the doped cathode host significantly, cf. eq. (2), which is why a suppression of the combination of localized holes is desirable to preserve a metastable reference state. This effect is more pronounced the 
stronger the stabilization of the host through oxygen dimerization is, which is why the heavily dimer-stabilized $\mathrm{Mg}$-doped $\mathrm{V}_{2} \mathrm{O}_{5}$ system shows only moderate voltage enhancement, while potentials are significantly improved for the Al- and Ti-doped systems as can be seen in Table 1. The voltage increase for the Li adsorption at Tidoped $\mathrm{V}_{2} \mathrm{O}_{5}$ is $1.47 \mathrm{~V}$, close to the expected improvement due to the 'bridging' of the band gap which was calculated as $1.97 \mathrm{eV}$ for the pristine vanadium pentoxide monolayer. Voltages beyond the low-concentration (single-atom adsorption) case were further calculated for the single-hole doping case (Ti), and the full voltage-capacity curves are shown in Figure 3. It is not surprising that the voltage profile of the doped $\mathrm{V}_{2} \mathrm{O}_{5}$ monolayer mostly follows the one of the pristine case, since the main

(a)
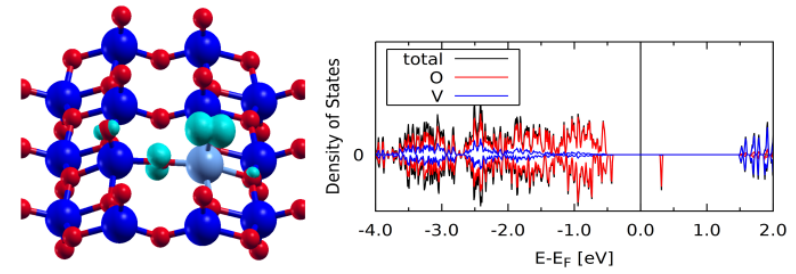

(b)

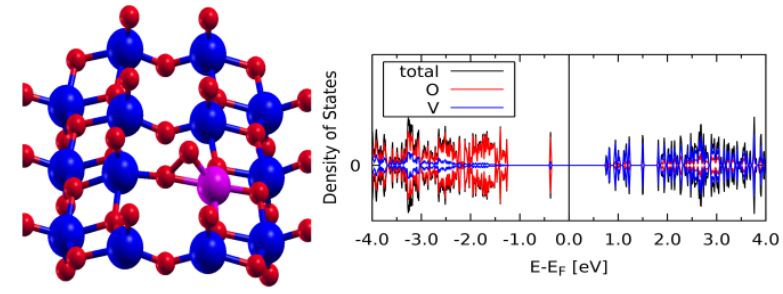

(c)

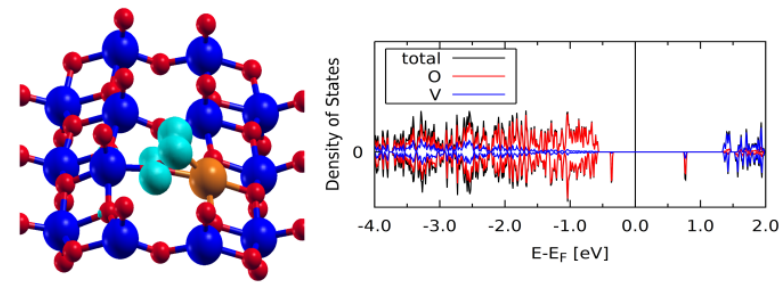

Figure 6. Spin densities (left) and densities of states (right) of (a) $\mathrm{Ti}_{0.17} \mathrm{~V}_{1.83} \mathrm{O}_{5}$, (b) $\mathrm{Al}_{0.17} \mathrm{~V}_{1.83} \mathrm{O}_{5}$, and (c) $\mathrm{Mg}_{0.17} \mathrm{~V}_{1.83} \mathrm{O}_{5}$. Up-spin density is represented in light blue, down-spin density in yellow (isosurface value $\left.0.005 e / \AA^{-3}\right)$, while vanadium, oxygen, titanium, aluminium, and magnesium atoms as blue, red, light blue, violet, and orange spheres, respectively. Up- and down-spin densities of states are shown with positive and negative sign, respectively; the vanadium-projected density of states is plotted in blue, oxygen-projected in red, and the full one in black. 
stabilization at low concentrations stems from the occupation of low-lying hole states, which are not available anymore once electrons are transferred from the adsorbate. Reduced electron-electron interactions, lattice deformations by doping and other substitution-related effects on the other hand do not lead to an obvious voltage change trend at higher metalation degrees. In accordance to existing literature, the $n$-hole $(n \geq 2)$ induced oxygen dimerization is not restricted to $\mathrm{V}_{2} \mathrm{O}_{5}$, but can be also observed for other TMO systems with an $\mathrm{O} p$ dominated valence band maximum. We have further calculated low-concentration insertion voltages with Li- (three holes), and Aldoped (one hole) brookite-type $\mathrm{TiO}_{2}(\mathrm{Pbca})$, as well as Al- (three holes), and V-doped (one hole) $\alpha-\mathrm{MoO}_{3}$ (Pnma) with the above elements ( $\mathrm{Li}, \mathrm{Mg}$, and Al). We found oxygen dimer formation in the investigated three-hole cases, along with consistently larger voltages for the doped TMOs compared to the pristine ones, similar to $\mathrm{V}_{2} \mathrm{O}_{5}$. Details on computed potentials and structures of doped $\mathrm{TiO}_{2}$ and $\mathrm{MoO}_{3}$ are presented in section 3 of the Supplementary Information.

\section{Suppression of Oxygen Dimer Formation and Mott Insulators}

The band gap in semiconducting and insulating TMOs can be either classified as charge-transfer or Mott-insulating type, depending on the character of valence band maximum and conduction band minimum states. In the TMOs discussed in the previous section, $\mathrm{V}_{2} \mathrm{O}_{5}, \mathrm{TiO}_{2}$ and $\mathrm{MoO}_{3}$, the $\mathrm{TM}$ species is formally fully oxidized, hence hybridization with TM $d$ orbitals is low in the valence band states and the $\mathrm{O} p$ character dominates, while the lowest unoccupied states are mostly of TM $d$ type. These charge-transfer insulators (semiconductors) do not necessarily need fully oxidized TM centers, but can also have occupied $d$ states at energies lower than the highest occupied $\mathrm{O} p$ states. In both cases, hole states introduced by p-doping are localized on oxygen centers, from which oxygen dimers can emanate. As previously discussed, their formation can restrict the gain in binding energies of metal atoms in the host and therefore achievable voltages, and the suppression of dimerization in $\mathrm{p}$ doped TMOs can help to retain more of the band structure component energy increase. Since the stabilization of the $\mathrm{O}$ dimer anions stems from the lowering of electronic state energies, it can be possibly counteracted by an increased energy penalty on the dimer polaron formation due to lattice strain. One example for such a strain-restricted p-doped TMO is the layered $R-3 m \mathrm{NiO}_{2}$. Two- and three-hole doping by $\mathrm{Mg}$ and $\mathrm{Li}$ 
(11 at\%), respectively, does not result in the formation of $\mathrm{O}_{2}{ }^{2-}$ or $\mathrm{O}_{2}{ }^{-}$anions, but rather localized O-centered holes at the valence band maximum, as shown in Figure 7(a) and (b). A similar resilience against $\mathrm{O}-\mathrm{O}$ dimerization was also found in the isostructural $R-3 m$ polymorph of $\mathrm{TiO}_{2}$.

To understand the different behavior of the p-doped $R-3 m$ structures, the energy increase upon lattice deformation has been computed in comparison to compounds exhibiting dimer formation. To this end, two oxygen centers bound to the same TM ion have been gradually rotated around it, decreasing the O-TM-O angle and hence the separation of the two oxide ions, while all other parameters have been held fixed. To rule out the effect of different dopants, and since we are only interested in the qualitative comparison among different systems, the computations have been performed on the pristine TMOs instead of the doped ones. As can be seen in Figure 7(c), the layered trigonal systems show a significantly higher strain energy (around two to three times higher at a typical $\mathrm{O}_{2}^{-}$anion bond length distance) at same magnitudes of oxide ion separation, indicating that indeed geometrical constraints are a probable reason for the suppressed dimer-polaron formation, since each oxide ion has two more adjacent $\mathrm{Ni}$ (or Ti) centers whose coordination polyhedra are distorted by the displaced anions as well. The coordination octahedra in the $R-3 m$ structures are all edge-connected, while the polyhedron connectivity is lower in the investigated structures that exhibit double-hole-polaron induced oxygen dimerization. For $\mathrm{NiO}_{2}$ with its relatively small $\mathrm{Ni}(\mathrm{IV})$ ions, the Li-doped structures are metastable and a $c$ shift of the dopant into a three-fold coordinated position is favored. The same holds for Li-doped trigonal $\mathrm{TiO}_{2}$, while the un-rearranged $\mathrm{Mg}$-doped structures of trigonal titanium and nickel dioxide remain more stable. However, adsorption of $\mathrm{Li}, \mathrm{Mg}$ and $\mathrm{Al}$ leads in all cases to large structural changes and dopant shifts, which makes the distinction between binding energy increase due to hole occupation on one hand and structural rearrangement (e.g. due to increasing cation-cation distances) on the other hand tedious. Nevertheless, the computed low-concentration voltage increases for $\mathrm{Mg}$ doped $R-3 m \mathrm{TiO}_{2}$ have been found to be significant, ranging from additional $2.80 \mathrm{~V}$ for $\mathrm{Mg}$ to $2.35 \mathrm{~V}$ higher values for $\mathrm{Li}$, and a $1.96 \mathrm{~V}$ increase for $\mathrm{Al}$, respectively, compared to a computed band gap of $2.48 \mathrm{eV}$, indicating especially for $\mathrm{Mg}$ the importance of further effects beyond the lowered work function of the material. However, the previously established trend of a beneficial effect of p-doping (without 
(a)

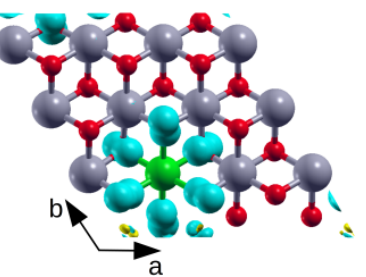

(b)

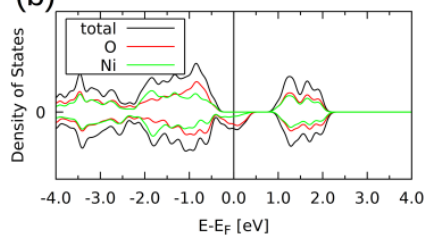

(c)

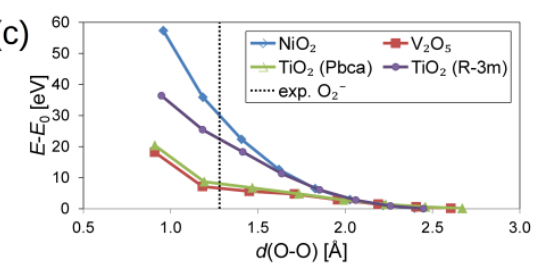

Figure 7. (a) Spin density, and (b) density of states of $\mathrm{Li}_{0.11} \mathrm{Ni}_{0.89} \mathrm{O}_{2}$, aa well as (c) deformation energy of $\mathrm{NiO}_{2}$. Up-spin density is represented in light blue, down-spin density in yellow (isosurface value 0.005 $\left.e / \AA^{-3}\right)$, while nickel, oxygen, and lithium atoms as grey, red, and green spheres, respectively. Up- and down-spin densities of states are shown with positive and negative sign, respectively; the nickel-projected density of states is plotted in green, oxygen-projected in red, and the full one in black. Energy values are given relative to the undeformed host and plotted against O-O distances, while the TM-O distances are kept constant. Deformation energies are also shown for trigonal and brookite-type $\mathrm{TiO}_{2}$, as well as $\alpha-\mathrm{V}_{2} \mathrm{O}_{5}$ for comparison.

oxygen dimerization) also holds for these systems. For details on structures, binding energies and voltages computed with the trigonal TMOs, see section 4 of the Supplementary Information.

Besides geometric constraints, oxygen dimerization is also not expected to occur in materials with TM $d$-dominated valence band maximum, since no localized oxygen hole states are expected in these to begin with. We chose monoclinic vanadium dioxide $\mathrm{VO}_{2}(\mathrm{M} 1)$ as a test system for a Mott-type insulator with a $d$ - $d$ band gap computed as $0.27 \mathrm{eV}$ (exp.: $0.6 \mathrm{eV}^{47}$ ). Substitutional doping with $\mathrm{Al}, \mathrm{Mg}$, and $\mathrm{Li}$ causes the formation of one, two and three holes, respectively. Densities of states of the $\mathrm{M}_{0.03} \mathrm{~V}_{0.97} \mathrm{O}_{2}$ system ( $\left.M=\mathrm{Al}, \mathrm{Mg}, \mathrm{Li}\right)$ are shown in Figure $8(\mathrm{~b})-(\mathrm{d})$. As expected, localized $d$ holes are created on vanadium centers around the dopant, resulting in a vanishing spin polarization of the respective atoms in the otherwise 
antiferromagnetically ordered $\mathrm{VO}_{2}(\mathrm{M} 1)$. Consistently with the number of introduced holes, two and three unpolarized centers are found in $\mathrm{Mg}$ - and Li-doped $\mathrm{VO}_{2}$, respectively; the corresponding spin densities are shown in section 5 of the Supplementary Information. For single-hole doped $\mathrm{Al}_{0.03} \mathrm{~V}_{0.97} \mathrm{O}_{2}$ two unpolarized vanadium centers are created as well, while additional spin polarization and charge is added to other centers, resulting in the 'self-donated' occupied mid-gap state in the DOS in Figure 8(b). The strong splitting of occupied and unoccupied TM $d$ states leads to the creation of hole states close to the conduction band minimum, but the lowenergy valence band maximum states are restored once electrons are donated from an inserted atom to the V-localized holes, as can be seen in section 5 of the Supplementary Information. Insertion potentials have been computed with respect to an occupied octahedral insertion site for all cases. For close dopant and solute, a binding energy and voltage increase is computed for $\mathrm{Mg}$-doped $\mathrm{VO}_{2}(\mathrm{M} 1)$ as an example case with inserted $\mathrm{Li}, \mathrm{Mg}$, and $\mathrm{Al}$, as shown in Table 2 (for a discussion of the effects of larger dopant-solute separation, see section 5 of the Supplementary Information). The voltage enhancements are not as large as the ones above, but bearing in mind the comparatively small band gap of $\mathrm{VO}_{2}(\mathrm{M} 1)$, which is also underestimated with our PBE setup, still distinct for the multivalent metals. Although the doped monoclinic vanadium dioxide phase is not expected to be a practical cathode material with a competitive voltage, the computed potential increase serves as a proof of concept that substitutional p-doping is also an effective strategy in combination with Mott-type insulators to improve achievable voltages with multivalent metals. 
(a)

(c)

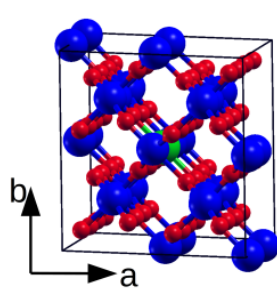

(c)
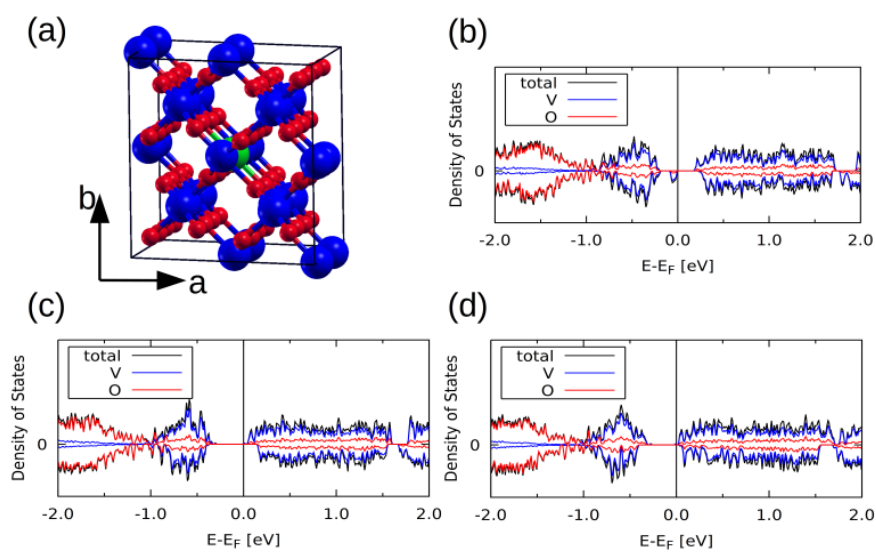

(d)

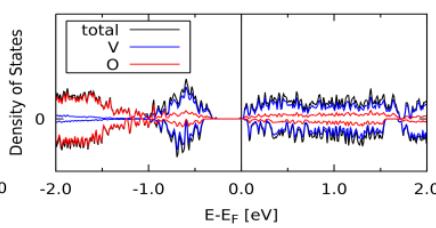

Figure 8. (a) Structure of doped $\mathrm{VO}_{2}(\mathrm{M} 1)$; vanadium, oxygen, and dopant atoms are represented as blue, red, and green spheres, respectively. Densities of states of (b) $\mathrm{Al}_{0.03} \mathrm{~V}_{0.97} \mathrm{O}_{2}$, (c) $\mathrm{Mg}_{0.03} \mathrm{~V}_{0.97} \mathrm{O}_{2}$, and (d) $\mathrm{Mg}_{0.03} \mathrm{~V}_{0.97} \mathrm{O}_{2}$ are shown with up- and down-spin densities of states having a positive and negative sign, respectively. The vanadium-projected density of states is plotted in blue, oxygen-projected in red, and the full one in black.

\section{Conclusions}

In this work, we have theoretically established the positive effect of substitutional pdoping on voltages in secondary batteries with insertion-type TMO electrodes. This is particularly interesting for multivalent metal ion batteries, as these are often prone to low voltages due to the larger number of charge carriers transferred per migrating ion. Lowering the TMO work function helps to alleviate this effect by strengthening the solute-host binding. The following general conclusions can be drawn for p-doped TMOs as electrode materials:

Table 2. Computed insertion voltages of $\mathrm{Li}, \mathrm{Mg}$, and $\mathrm{Al}$ in pristine and $\mathrm{Mg}$-doped $\mathrm{VO}_{2}(\mathrm{M} 1)$ against the respective bulk metals.

\begin{tabular}{lll}
\hline \multirow{2}{*}{ adsorbate } & $\mathrm{VO}_{2}(\mathrm{Ml})$ & $\mathrm{Mg}_{0.03} \mathrm{~V}_{0.97} \mathrm{O}_{2}(\mathrm{Ml})$ \\
$\mathrm{Li}$ & $\mathrm{U}[\mathrm{V}]$ & $\mathrm{U}[\mathrm{V}]$ \\
$\mathrm{Mg}$ & 2.12 & 2.14 \\
$\mathrm{Al}$ & 1.30 & 1.43 \\
& 1.49 & 1.56 \\
\hline
\end{tabular}


1. Cation binding energy is strengthened by up to the size of the band gap, confirming that band structure modulation induced by doping is the main factor for the potential enhancement. A consistent voltage increase is observable as long as unoccupied hole states exist, beyond that no improvement is expected in general. For higher voltages at a large capacity, the dopant concentrations need to be raised accordingly.

2. For strongly correlated TMOs with an $\mathrm{O} p$-dominated valence band maximum, oxygen dimerization can occur with dopants inducing more than a single hole state. The dimerization leads to a stabilization of the unmetalated electrode material, decreasing the maximum gain in binding energy and hence voltages (for TMO cathodes). Depending on the degree of stabilization, the formed dimers can possibly persist upon metalation, further reducing the number of available hole states.

3. Oxygen dimer formation is suppressed in compounds with sterically restricted oxide ion movement due to occurring lattice strains related to the dimer polaron formation, and voltage gains close to the theoretical limit of the band gap width are expected (excluding other effects like lattice reconstruction etc.).

4. Mott-type insulators do not exhibit oxygen dimer formation due to the localization of holes on the TM centers.

5. Large electron-polaron hopping barriers can suppress the electron-hole combination for large dopant-solute separations, which might occur in e.g. unevenly doped TMOs with low ionic diffusivities.

We hope that these fundamental insights from electronic structure computations of p-doped TMO materials can help guide a rational design of these compounds in the future, in particular for a possible application in post-Li ion batteries, and inform prospective theoretical and experimental research in this field.

\section{Acknowledgements}

This work was supported by the Ministry of Education of Singapore (Grant No. MOE2015-T2-1-011). 


\section{References}

1. Mineral Commodity Summaries 2019, U.S. Department of the Interior, Reston, VA, 2019.

2. M. S. Whittingham, C. Siu and J. Ding, Acc. Chem. Res., 2018, 51, 258-264.

3. S. P. Ong, L. Wang, B. Kang and G. Ceder, Chem. Mater., 2008, 20, 1798-1807.

4. A. Jain, S. P. Ong, G. Hautier, W. Chen, W. D. Richards, S. Dacek, S. Cholia, D. Gunter, D. Skinner, G. Ceder and K. A. Persson, APL Mater., 2013, 1, 011002.

5. A. Jain, G. Hautier, S. P. Ong, C. J. Moore, C. C. Fischer, K. A. Persson and G. Ceder, Phys. Rev. B, 2011, 84, 045115.

6. D. F. C. Morris and E. L. Short, Nature, 1969, 224, 950-952.

7. D. Koch and S. Manzhos, J. Phys. D: Appl. Phys., 2019, accepted manuscript.

8. J. Lüder, F. Legrain, Y. Chen and S. Manzhos, MRS Commun., 2017, 7, 523-540.

9. F. Legrain and S. Manzhos, J. Power Sources, 2015, 274, 65-70.

10. F. Legrain and S. Manzhos, J. Chem. Phys., 2017, 146, 034706.

11. M. Panagopoulou, D. Vernardou, E. Koudoumas, N. Katsarakis, D. Tsoukalas and Y. S. Raptis, J. Phys. Chem. C, 2017, 121, 70-79.

12. J. Liu, H. Xia, D. Xue and L. Lu, J. Am. Chem. Soc., 2009, 131, 12086-12087.

13. D. B. Le, S. Passerini, F. Coustier, J. Guo, T. Soderstrom, B. B. Owens and W. H. Smyrl, Chem. Mater., 1998, 10, 682-684.

14. G. Gershinsky, H. D. Yoo, Y. Gofer and D. Aurbach, Langmuir, 2013, 29, 1096410972.

15. G. Ali, J. H. Lee, S. H. Oh, B. W. Cho, K.-W. Nam and K. Y. Chung, ACS Appl. Mater. Interfaces, 2016, 8, 6032-6039.

16. S. Suthirakun, S. Jungthawan and S. Limpijumnong, J. Phys. Chem. C, 2018, 122, 5896-5907.

17. D. Ni, J. Shi, W. Xiong, S. Zhong, B. Xu and C. Ouyang, Phys. Chem. Chem. Phys., 2019, 21, 7406-7411.

18. T. Wu, K. Zhu, C. Qin and K. Huang, J. Mater. Chem. A, 2019, 7, 5612-5620.

19. K. West, B. Zachau-Christiansen, M. J. L. Østergård and T. Jacobsen, J. Power Sources, 1987, 20, 165-172.

20. X. Rui, Z. Lu, H. Yu, D. Yang, H. H. Hng, T. M. Lim and Q. Yan, Nanoscale, 2013, $5,556-560$. 
21. X. Zhao, X. Zhang, D. Wu, H. Zhang, F. Ding and Z. Zhou, J. Mater. Chem. A, 2016, 4, 16606-16611.

22. Z. Wang, Q. Su and H. Deng, Phys. Chem. Chem. Phys., 2013, 15, 8705-8709.

23. J. Cheng, B. Wang, H. L. Xin, G. Yang, H. Cai, F. Nie and H. Huang, J. Mater. Chem. A, 2013, 1, 10814-10820.

24. J. P. Perdew, K. Burke and M. Ernzerhof, Phys. Rev. Lett., 1996, 77, 3865-3868.

25. J. P. Perdew, K. Burke and M. Ernzerhof, Phys. Rev. Lett., 1997, 78, 1396-1396.

26. J. M. Soler, E. Artacho, J. D. Gale, A. García, J. Junquera, P. Ordejón and D. Sánchez-Portal, J. Phys.: Condens. Matter, 2002, 14, 2745-2779.

27. F. Legrain, O. Malyi and S. Manzhos, J. Power Sources, 2015, 278, 197-202.

28. D. Koch and S. Manzhos, MRS Adv., 2019, 4, 837-842.

29. J. Junquera, Ó. Paz, D. Sánchez-Portal and E. Artacho, Phys. Rev. B, 2001, 64, 235111 .

30. E. Artacho, D. Sánchez-Portal, P. Ordejón, A. García and J. M. Soler, Phys. Status Solidi B, 1999, 215, 809-817.

31. E. Anglada, J. M. Soler, J. Junquera and E. Artacho, Phys. Rev. B, 2002, 66, 205101.

32. H. J. Monkhorst and J. D. Pack, Phys. Rev. B, 1976, 13, 5188-5192.

33. M. Methfessel and A. T. Paxton, Phys. Rev. B, 1989, 40, 3616-3621.

34. R. S. Mulliken, J. Chem. Phys., 1955, 23, 1841-1846.

35. A. Kokalj, J. Mol. Graphics Modell., 1999, 17, 176-179. Code available from http://www.xcrysden.org/.

36. A. Urban, D.-H. Seo and G. Ceder, Npj Comput. Mater., 2016, 2, 16002.

37. V. V. Kulish, D. Koch and S. Manzhos, Energies, 2017, 10.

38. M. A. Korotin, V. I. Anisimov, T. Saha-Dasgupta and I. Dasgupta, J. Phys.: Condens. Matter, 1999, 12, 113-124.

39. J. L. de Boer, A. Meetsma, J. Baas and T. T. M. Palstra, Phys. Rev. Lett., 2000, 84, 3962-3965.

40. S. Suthirakun, A. Genest and N. Rösch, J. Phys. Chem. C, 2018, 122, 150-157.

41. M. V. Mostovoy and D. I. Khomskii, Solid State Commun., 1999, 113, 159-163.

42. X. Ming, H.-G. Fan, Z.-F. Huang, F. Hu, C.-Z. Wang and G. Chen, J. Phys.: Condens. Matter, 2008, 20, 155203.

43. P. Canepa, G. Sai Gautam, D. C. Hannah, R. Malik, M. Liu, K. G. Gallagher, K. A. Persson and G. Ceder, Chem. Rev., 2017, 117, 4287-4341. 
44. P. VerNooy, Acta Crystallogr. Sect. C, 1993, 49, 433-434.

45. S. C. Abrahams and J. Kalnajs, Acta Crystallogr., 1955, 8, 503-506.

46. S. Chen and L.-W. Wang, Phys. Rev. B, 2014, 89, 014109.

47. H. W. Verleur, A. S. Barker and C. N. Berglund, Phys. Rev., 1968, 172, 788-798. 


\title{
Supplementary Information:
}

\section{Can Doping of Transition Metal Oxide Cathode Materials Increase Achievable Voltages with Multivalent Metals?}

\author{
Daniel Koch $^{\mathrm{a}}$ and Sergei Manzhos ${ }^{\mathrm{b}}$
}

a. Department of Mechanical Engineering, National University of Singapore, Block EA \#07-08, 9 Engineering Drive 1, Singapore 117576. Email: koch.danielm@gmail.com

b. Centre Énergie Matériaux Télécommunications, Institut National de la Recherche Scientifique, 1650 boulevard Lionel-Boulet, Varennes QC J3X1S2 Canada. Email: sergei.manzhos@emt.inrs.ca

\section{Adjustment of Pseudo-Atomic Orbital Energy Shifts}

Table S3. Comparison of experimentally obtained insertion voltages with computed ones using PAO energy shifts of $0.001 \mathrm{Ry}$ on $\mathrm{V}, \mathrm{Mo}$, Ti, and $\mathrm{O}$, while the PAO energy shifts for $\mathrm{Li}$ and $\mathrm{Mg}$ are adjusted to match the cohesive energies of the bulk metal.

\begin{tabular}{|l|l|l|}
\hline Phase Formed & Computed Voltage [V] & Experimental Voltage [V] \\
\hline $\mathrm{Mg}_{0.5} \mathrm{~V}_{2} \mathrm{O}_{5}$ & 2.29 & $2.3^{1}$ \\
\hline $\mathrm{Li}_{0.25} \mathrm{MoO}_{3}$ & 2.91 & $2.7^{2}$ \\
\hline $\mathrm{LiTiO}_{2}$ & 1.60 & $1.6^{3}$ \\
\hline
\end{tabular}




\section{Electron-Hole Separation in p-Doped $\mathrm{V}_{2} \mathrm{O}_{5}$ Nanosheets}

The comparison in Table $\mathrm{S} 4$ shows the case of $\mathrm{Li}$ and $\mathrm{Mg}$ adsorption on $\mathrm{Mg}$-doped $\mathrm{V}_{2} \mathrm{O}_{5}$. For cases in which the occupation of hole states by the donated electrons is energetically highly favorable, solute-dopant distances exceeding the chosen simulation cell size would likely be needed to prevent electron-hole combination, as already for e.g. $\mathrm{Li}$ on the Ti-doped $\mathrm{V}_{2} \mathrm{O}_{5}$ nanosheet electrons and holes always recombine in the employed cell.

Table S4. Spin densities and voltages (against bulk metal) of Li- and $\mathrm{Mg}$-adsorbed $\mathrm{Mg}_{0.17} \mathrm{~V}_{1.83} \mathrm{O}_{5}$ for minimal and large dopant-adsorbate distances. Up-spin density is represented in light blue, down-spin density in yellow (isosurface value $0.005 e / \AA^{-3}$ ), while vanadium, oxygen, titanium, and magnesium atoms are shown as blue, red, light blue, and orange spheres, respectively. For larger distances, an electron-hole separation and lower voltages are observed.

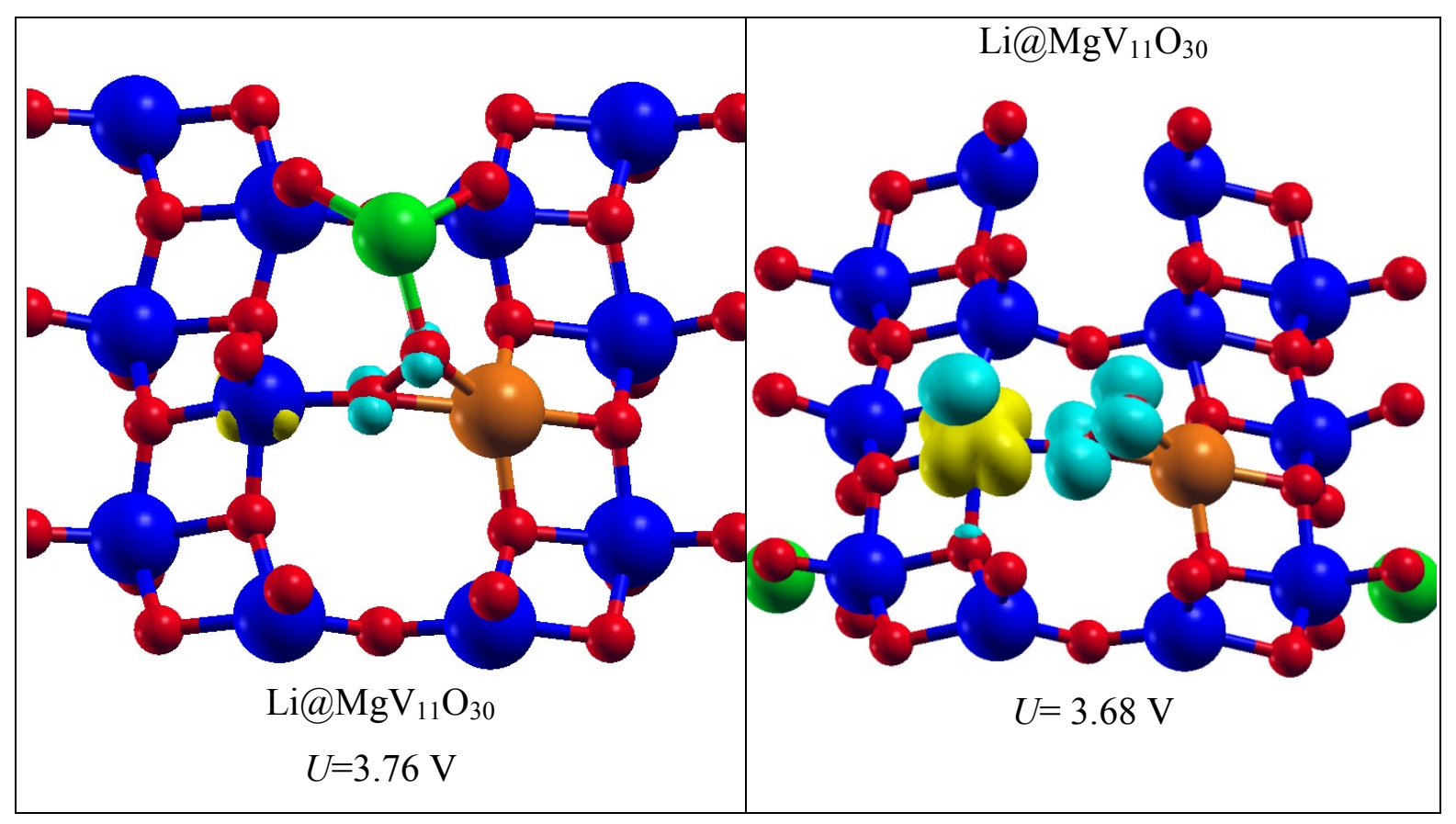




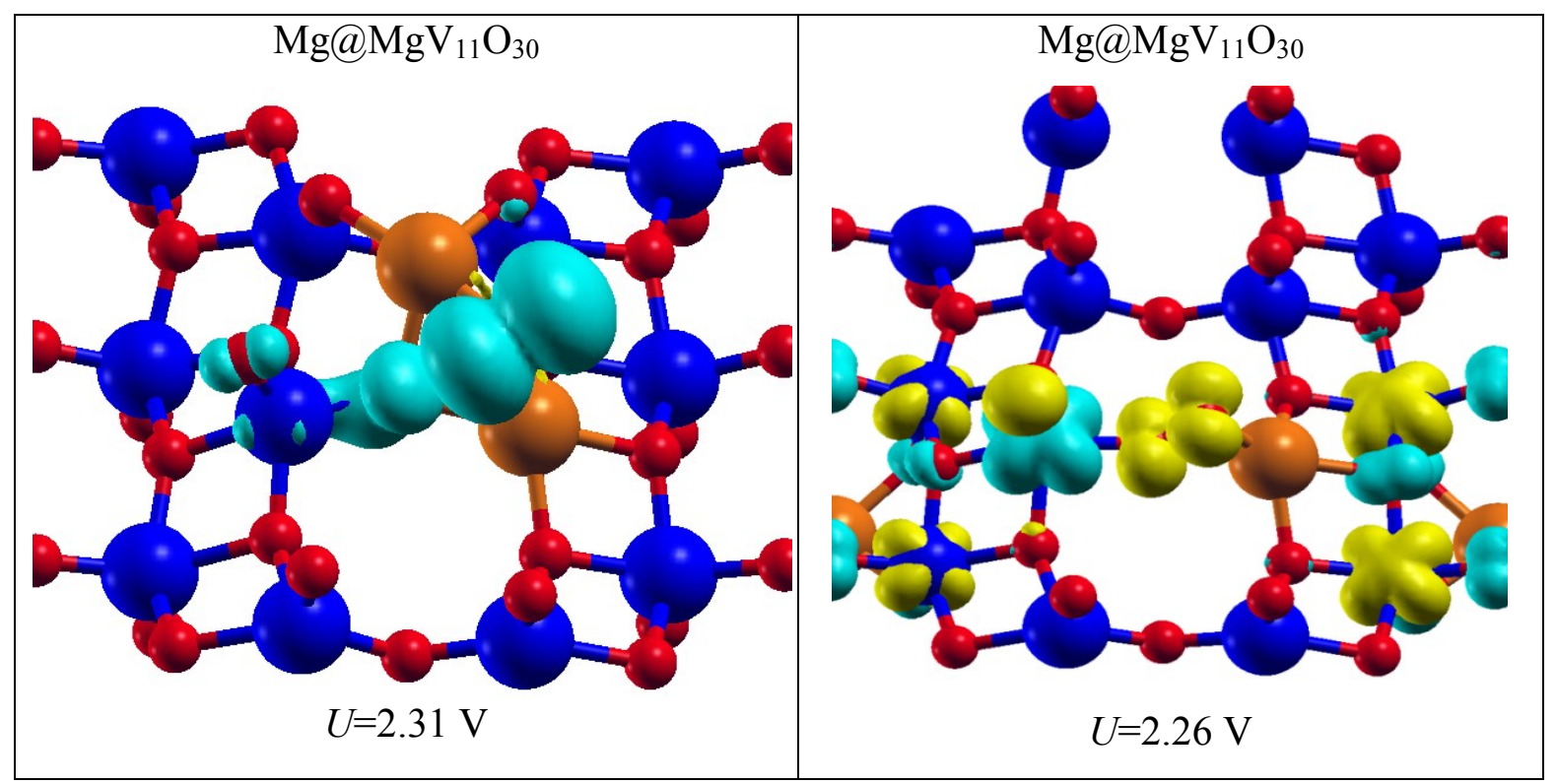

\section{Structures and Voltages of/with P-Doped $\alpha-\mathrm{MoO}_{3}$ and Brookite $\mathrm{TiO}_{2}$}

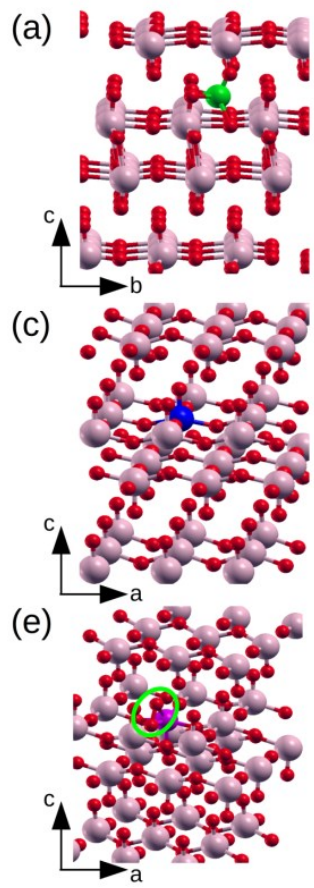

(b)

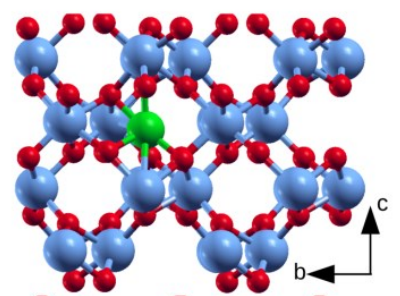

(d)

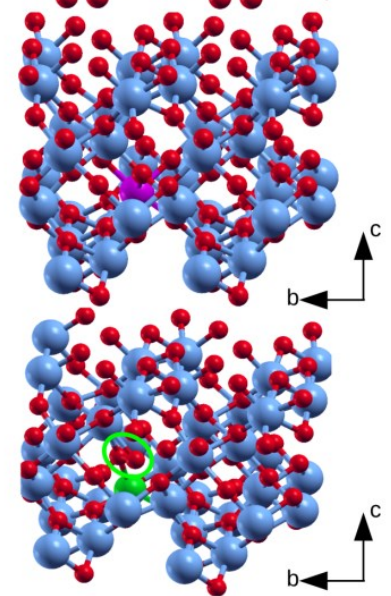

Figure S9. Crystal structures of $\alpha-\mathrm{MoO}_{3}$ (left column) and brookite $\mathrm{TiO}_{2}$ (right column) with preferred stable insertion site $(\mathrm{a}, \mathrm{b})$, single-hole dopant (c: V, d: Al) and triple-hole dopant (e: Al, f: Li). Molybdenum centers are represented in light red, titanium in light blue, oxygen in red, vanadium in blue, aluminium in purple, and lithium 
in green. Oxygen dimers are circled in green for better visibility.

The computed voltages in Table S5 show a potential increase for the single-hole doped $\mathrm{MoO}_{3}$ and $\mathrm{TiO}_{2}$ phases. The magnitude of the increase is the largest for the insertion of $\mathrm{Li}$ and smaller for $\mathrm{Mg}$ and $\mathrm{Al}$. The lithiated $\mathrm{V}$-doped $\mathrm{MoO}_{3}$ shows a voltage increase of more than $2.0 \mathrm{~V}$ although the computed $\mathrm{MoO}_{3}$ band gap is only $1.79 \mathrm{eV}$ (brookite $\mathrm{TiO}_{2}: 2.05 \mathrm{eV}$ ), which indicates further factors for the potential enhancement than the band structure energy (e.g. decreased lattice rigidity). As expected, the oxygen-dimerized triple-hole doped $\mathrm{MoO}_{3}$ shows a diminished voltage improvement due to the stabilization of the host by the formation of the dimer, even destabilizing the bonding for $\mathrm{Al}$ compared to the pristine phase. For $\mathrm{TiO}_{2}$ on the other hand, the dimerized structure shows further voltage enhancement with $\mathrm{Mg}$ and Al, which is probably caused by lattice distortion effects, since the Li dopant slightly changes its position into a tetrahedrally coordinated site, which might be energetically more favorable in the dimerized structure and indicating a metastable Li-doped host.

Table S5. Computed voltages for the $\mathrm{Li}, \mathrm{Mg}$, and $\mathrm{Al}$ insertion in pristine and single- or triple-hole doped $\alpha-\mathrm{MoO}_{3}\left(\mathrm{~V}\right.$ - and Al-doped, respectively) and brookite $\mathrm{TiO}_{2}$ (Al- and Li-doped, respectively).

\begin{tabular}{|c|c|c|c|c|c|c|}
\hline \multirow{2}{*}{ adsorbate } & \multicolumn{3}{|c|}{$\alpha-\mathrm{MoO}_{3}, U[\mathbf{U}]$} & \multicolumn{3}{c|}{ Brookite $\mathrm{TiO}_{2}, \boldsymbol{U}[\mathrm{V}]$} \\
\cline { 2 - 7 } & pristine & V-doped & Al-doped & pristine & Al-doped & Li-doped \\
\hline $\mathbf{L i}$ & 2.61 & 4.64 & 3.15 & 1.65 & 2.86 & 2.09 \\
\hline $\mathbf{M g}$ & 1.70 & 2.67 & 1.71 & 0.76 & 1.41 & 2.19 \\
\hline $\mathbf{A l}$ & 1.67 & 1.87 & 1.43 & 0.71 & 1.10 & 2.31 \\
\hline
\end{tabular}




\section{Structures and Voltages of/with P-Doped Trigonal $\mathrm{NiO}_{2}$ and $\mathrm{TiO}_{2}$}

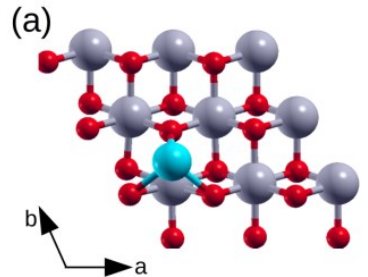

(c)

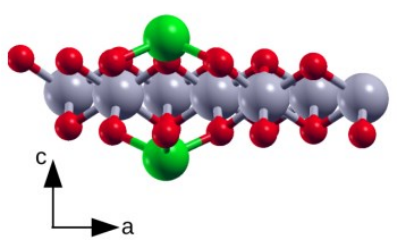

(e)

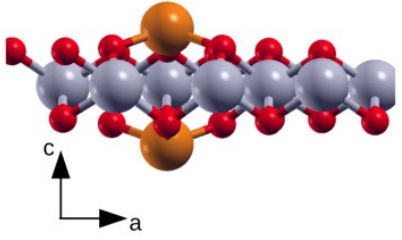

(b)

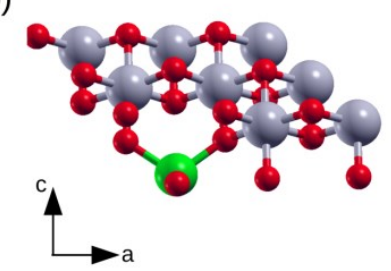

(d)

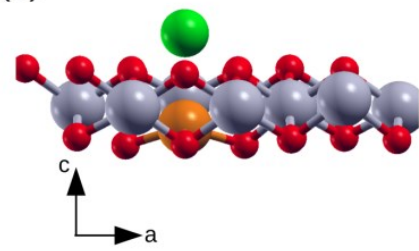

(f)

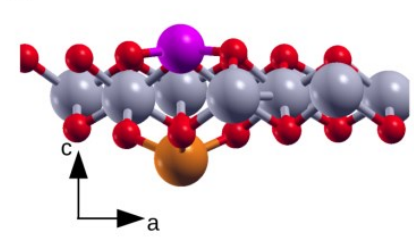

Figure S10. (a) Stable cationic adsorption site on $R-3 m$ $\mathrm{NiO}_{2}$ and $\mathrm{TiO}_{2}$ nanosheets (turquoise), (b) preferential position of a $\mathrm{Li}$ dopant in $\mathrm{NiO}_{2}$ and $\mathrm{TiO}_{2}$, (c) structure of Li-adsorbed Li-doped trigonal $\mathrm{NiO}_{2}$ and $\mathrm{TiO}_{2}$, (d) structure of $\mathrm{Li}$-adsorbed $\mathrm{Mg}$-doped (or vice versa) trigonal $\mathrm{NiO}_{2}$ and $\mathrm{TiO}_{2}$, (e) structure of Mg-adsorbed Mg-doped trigonal $\mathrm{NiO}_{2}$ and $\mathrm{TiO}_{2}$, (f) structure of Aladsorbed $\mathrm{Mg}$-doped trigonal $\mathrm{NiO}_{2}$ and $\mathrm{TiO}_{2}$. Nickel or titanium centers are represented in grey, oxygen in red, lithium in green, magnesium in orange and aluminium in purple.

Table S6. Computed voltages for the $\mathrm{Li}, \mathrm{Mg}$, and $\mathrm{Al}$ adsorption on a pristine and double-hole (Mg-) doped trigonal $\mathrm{NiO}_{2}$ and $\mathrm{TiO}_{2}$ nanosheet.

\begin{tabular}{|c|c|c|c|c|}
\hline \multirow{2}{*}{ adsorbate } & \multicolumn{2}{|c|}{$\boldsymbol{R - 3 m} \mathbf{N i O}_{2}, \boldsymbol{U}[\mathbf{V}]$} & \multicolumn{2}{c|}{$\boldsymbol{R}-3 m \mathbf{T i O}_{2}, \boldsymbol{U}[\mathbf{V}]$} \\
\cline { 2 - 5 } & pristine & Mg-doped & pristine & Mg-doped \\
\hline $\mathbf{L i}$ & 2.83 & 4.18 & 2.18 & 4.52 \\
\hline $\mathbf{M g}$ & 1.49 & 2.75 & 0.65 & 3.46 \\
\hline $\mathbf{A l}$ & 1.57 & 2.32 & 0.12 & 2.08 \\
\hline
\end{tabular}




\section{Electron-Hole Separation in P-Doped $\mathrm{VO}_{2}$}

Figure S11 shows the spin densities for one-, two-, and three-hole doped $\mathrm{VO}_{2}(\mathrm{M} 1)$ with localized hole states carrying no spin magnetic moment. Similar to what has been previously established in the case of $\mathrm{V}_{2} \mathrm{O}_{5}$ in the main text, restricted electron mobility can prevent the combination of localized electrons and holes if the inserted species is separated too far from the dopant and the energy penalty for electron hopping exceeds the gain in recombination energy.

(a)

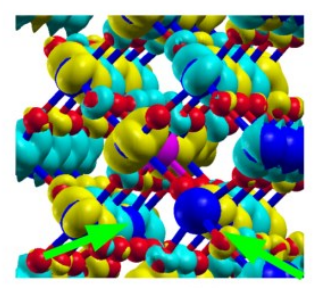

(b)

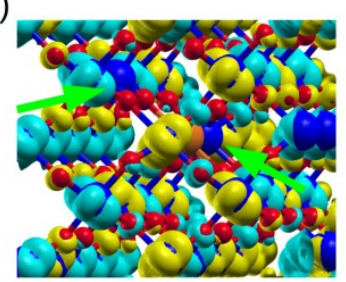

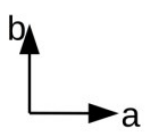

(c)

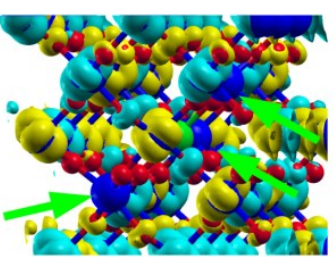

Figure S11. Spin densities of (a) $\mathrm{Al}_{0.03} \mathrm{~V}_{0.97} \mathrm{O}_{2}$, (b) $\mathrm{Mg}_{0.03} \mathrm{~V}_{0.97} \mathrm{O}_{2}$, and (c) $\mathrm{Mg}_{0.03} \mathrm{~V}_{0.97} \mathrm{O}_{2}$. Up-spin density is represented in light blue, down-spin density in yellow (isosurface value $0.01 \mathrm{e} / \AA^{-3}$ ), while vanadium, oxygen, aluminium, magnesium, and lithium atoms as blue, red, light blue, violet, orange, and green spheres, respectively. Green arrows indicate the positions of the spinunpolarized vanadium atoms for better visibility.

As shown in Figure $\mathrm{S} 12$, insertion of $\mathrm{Mg}$ further away from the dopant site results in lower voltages and the preservation of more hole states. While for configuration 1 (close) only one hole remains, as can be seen from the spin density and the narrower spin-up density of states above the Fermi level, configuration 2 retains both hole states and two localized occupied mid-gap states. The retention of one hole state for the low-energy double-holedoped two-electron donated configuration 1 suggests that the small stabilization by electronhole combination (due to the small band gap, see main text) does not suffice to cause significant electron hopping even if the solute is close to the dopant. 


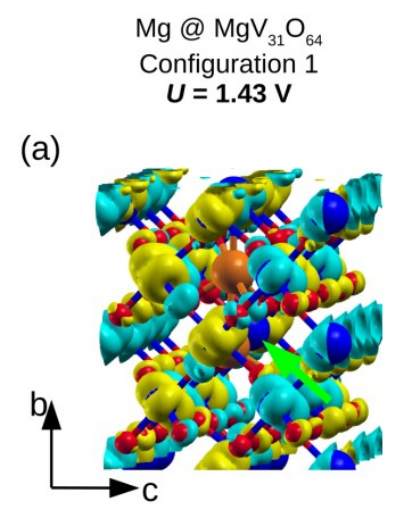

$\mathrm{Mg} @ \mathrm{MgV}_{31} \mathrm{O}_{64}$ Configuration 2

$U=1.36 \mathrm{~V}$

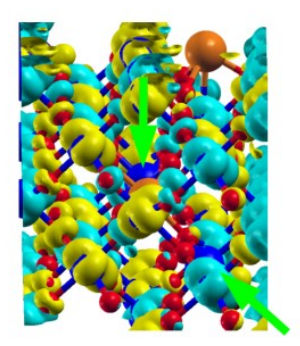

(b)
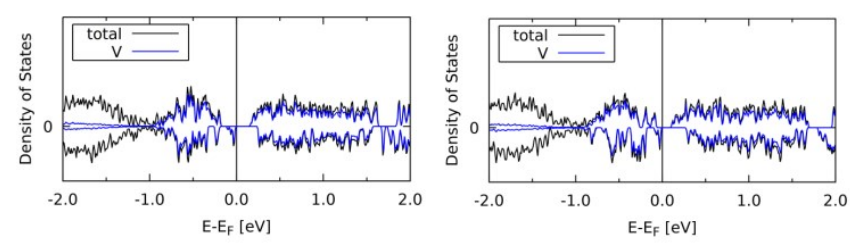

Figure S12. (a) Spin densities and (b) densities of state for different dopant-solute distances in $\mathrm{Mg}$-inserted $\mathrm{Mg}$ doped $\mathrm{VO}_{2}(\mathrm{M} 1)$. In the left column, the solute and dopant are in proximity, in the right column they are further separated. Insertion voltages are given on the top of the respective column. Vanadium centers are represented in blue, oxygen in red, and magnesium in orange. Up-spin density is represented in light blue, down-spin density in yellow (isosurface value $0.01 \AA^{-3}$ ). Up- and down-spin densities of states are shown with positive and negative sign, respectively; the vanadium-projected density of states is plotted in blue, the full one in black. Green arrows indicate the positions of the spin-unpolarized vanadium atoms for better visibility.

\section{References}

1. G. Gershinsky, H. D. Yoo, Y. Gofer and D. Aurbach, Langmuir, 2013, 29, 10964-10972.

2. U. K. Sen and S. Mitra, RSC Adv., 2012, 2, 11123-11131.

3. M. Anji Reddy, V. Pralong, U. V. Varadaraju and B. Raveau, Electrochem. Solid-State Lett., 2008, 11, A132-A134. 